\title{
Weak Target Detection within the Nonhomogeneous Ionospheric Clutter Background of HFSWR Based on STAP
}

\author{
Xin Zhang, Qiang Yang, and Weibo Deng \\ School of Electronics Information Engineering, Harbin Institute of Technology, Harbin 150001, China \\ Correspondence should be addressed to Weibo Deng; dengweibo@hit.edu.cn
}

Received 1 April 2013; Revised 28 July 2013; Accepted 7 August 2013

Academic Editor: Ulrich Nickel

Copyright ( 2013 Xin Zhang et al. This is an open access article distributed under the Creative Commons Attribution License, which permits unrestricted use, distribution, and reproduction in any medium, provided the original work is properly cited.

\begin{abstract}
High Frequency Surface Wave Radar (HFSWR) can perform the functions of ocean environment monitoring, target detection, and target tracking over the horizon. However, its system's performance is always limited by the severe ionospheric clutter environment, especially by the nonhomogeneous component. The nonhomogeneous ionospheric clutter generally can cover a few Doppler shift units and a few angle units. Consequently, weak targets masked by the nonhomogeneous ionospheric clutter are difficult to be detected. In this paper, a novel algorithm based on angle-Doppler joint eigenvector which considers the angle-Doppler map of radar echoes is adopted to analyze the characteristics of the nonhomogeneous ionospheric clutter. Given the measured data set, we first investigate the correlation between the signal of interest (SOI) and the nonhomogeneous ionospheric clutter and then the correlation between the nonhomogeneous ionospheric clutters in different two ranges. Finally, a new strategy of training data selection is proposed to improve the joint domain localised (JDL) algorithm. Simulation results show that the improved-JDL algorithm is effective and the performance of weak target detection within nonhomogeneous ionospheric clutter is improved.
\end{abstract}

\section{Introduction}

HFSWR exploits the surface wave mode of vertical polarization electromagnetic wave propagating over the sea water to detect ships and aircrafts at distances beyond the line of sight. It has drawn much attention in recent years for its notable features of large scale, long distance, and all-day adaptability. In general, the factors which affect the performance of HFSWR are the sea clutter, ionospheric clutter, radio interference, and noise, among which, the key factor that determines targets detection performance is the ionospheric clutter [1]. The situation herein is very complex, since the motion states of the ionosphere are distinct at different temporal and spatial locations. Until now, a widely accepted model for the ionospheric clutter in HFSWR is still not found. It follows that how to suppress the ionospheric clutter and how to improve the weak target detection performance in HFSWR systems are hot topics worth further investigation.

In HFSWR systems, long coherent integration time is necessary for better detection performance and higher Doppler resolution. During this process, the state of the ionosphere is changing rapidly and irregularly, which leads to an obvious problem that the ionospheric clutter can cover a few Doppler shift units after the coherent integration. Therefore, it is difficult for the frequency domain adaptive matched filtering algorithm to detect the weak targets buried in the ionospheric clutter. On the other hand, both the beambroadening effect resulting from the smaller array aperture compared to the wavelength, and the regional characteristic of ionosphere can lead to the fact that ionospheric clutter always covers a broad angular region. Consequently, the ionospheric clutter is difficult to be suppressed either in the Doppler domain or in the angle domain.

Space-time adaptive processing (STAP) is proposed by Brennan and Reed in the 1970s and has become one of the major research directions around the world [2]. It has been mainly exploited to suppress homogeneous and nonhomogeneous clutters in airborne radar systems in the Dopplerangle domain [3-6]. It also has been applied in many other specific applications, both in military and civil fields, such as spaceborne radar, communication, sonar, navigation, and microphone array [7, 8]. In HFSWR systems, STAP has been adopted to counter the sea clutter in shipborne radar systems 
[9] and suppress the ionospheric clutter in shore-based radar systems.

Groups led by Fabrizio and Adve have begun to investigate the problem of ionospheric clutter suppression based on STAP and obtained a bulk of measured data [10-13]. In [13], Ravan et al. developed a new model to predict the radio wave propagation in ionosphere plasma density irregularities, and it turns out that their model fits the data gathered in Canada well. Ravan and Saleh et al. also analyzed a group of STAP algorithms such as joint domain localized, direct data domain, hybrid, PAMF, and further a new proposed fast algorithm FFA $[13,14]$. Joint domain localized (JDL) is a classical statistical STAP algorithm broadly used in airborne radar [15]. It features preferable low computational cost and high performance in homogeneous clutter suppression. Especially for the clutter with independent and identical distribution, JDL is highly effective. But in HFSWR, the ionospheric clutter is nonhomogeneous. It is always difficult to obtain enough training data for JDL. Direct data domain (D3), hybrid, and PAMF are classical STAP algorithms for nonhomogeneous clutter in airborne radar systems. These algorithms suffer from high computational cost and poor real-time performance in HFSWR due to the long coherent integration time (CIT) and large space-time dimension. FFA is also a low computational cost algorithm, but how to divide the space-time region is still under investigation.

In this paper, we first analyze the correlation between the SOI and the nonhomogeneous ionospheric clutter; and then, the correlation between the nonhomogeneous ionospheric clutters at two different ranges has been analyzed. On this basis, we propose a new strategy to select the training data set. By means of the reasonable training data selection, the nonhomogeneous ionospheric clutter can be suppressed more effectively. Meanwhile, weak targets in the direction of ionospheric can also be detected.

In Section 2, we first analyze the correlation including the correlation between the SOI and the nonhomogeneous ionospheric clutter and the one between the nonhomogeneous ionospheric clutters at two different ranges. In Section 3, we proposed an improved-JDL algorithm according to the analysis results given in Section 2. Finally, the superiority of the improved-JDL algorithm is demonstrated via simulation based on practically measured data.

\section{Characteristic Analysis of Nonhomogeneous Ionospheric Clutter}

In HFSWR, the electromagnetic waves travel not only mainly over the sea surface but also partly into the sky due to nonidealities of the receiver antenna array. Under certain conditions, the electromagnetic waves emitted into the sky can be reflected by the ionosphere and then received by the nonideal receiver antenna array. This is how the ionospheric clutter emerges. The ionospheric clutter is very intricate as a result of the nonhomogeneous layered structure and the rapidly changing state of the ionosphere. For these reasons, ionospheric clutter suppression is a critical difficulty in HFSWR. The study of ionospheric clutter suppression

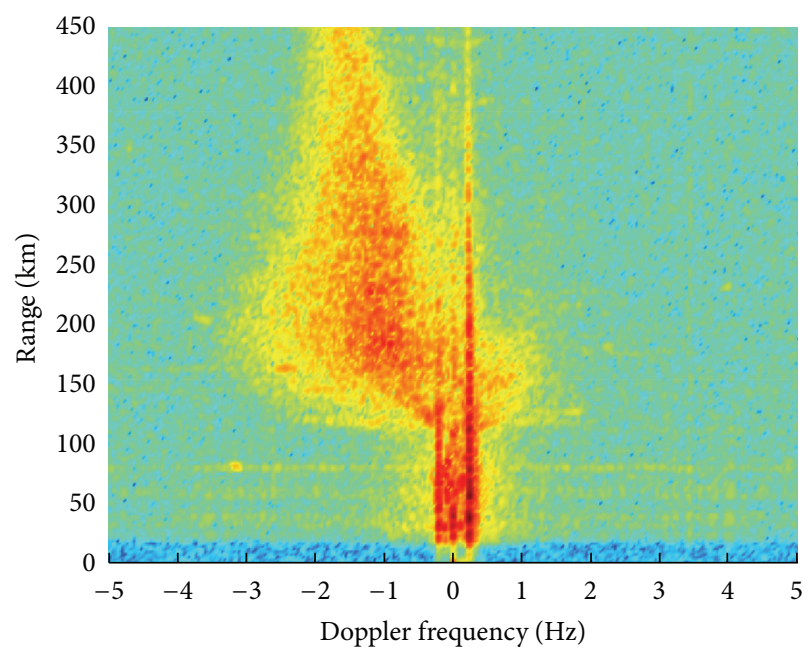

Figure 1: Range-Doppler Map.

depends heavily on the understanding of the characteristics of ionospheric clutter.

Firstly, we analyze the characteristics of the ionospheric clutter. The measured data is obtained through the HFSWR system in Weihai, China on May 12, 2012, and then processed by matched filters in range, Doppler, and digital beamforming in turn. A range-Doppler map in one beam is shown in Figure 1, and an angle-Doppler map in one range bin is shown in Figure 2.

In STAP, the data is processed on the basis of the angleDoppler map. In this case, characteristics of the nonhomogeneous ionospheric clutter should be analyzed based on the angle-Doppler map.

In Figure 2, the nonhomogeneous ionospheric clutter covers a large region of the angle-Doppler map. It does not make any sense to analyze such a broad region. So we divide the whole angle-Doppler region into small scales, and the characteristic analysis of the nonhomogeneous ionospheric clutter should also be in the light of the small local angleDoppler region. We call this small local region the AngleDoppler Local Region (ADLR). The analysis in Section 2 is solely concentrated on the concept of ADLR.

In Section 2.1, we have to decide what the appropriate size of ADLR is in order to make sure that the clutter in the ADLR is simple.

We suppose $\mathbf{X}_{l}$ is an ADLR sequence for $l=0,1,2, \ldots$ of independent range samples as discussed in the literature [2]. But the sample in one range bin may relate with some samples in other independent range bins.

In Section 2.2, we propose the correlation analyzing method based on the angle-doppler joint eigenvector (ADJE) of ADLR.

Based on the correlation analyzing method in Section 2.2 and the size of ADLR decided in Section 2.1, we analyze the SOI correlation in the noise environment (in Section 2.3) and in the ionospheric clutter environment (in Section 2.4).

In the last Section 2.5, we analyze the ionospheric clutter correlation between ADLRs in different range bins. 


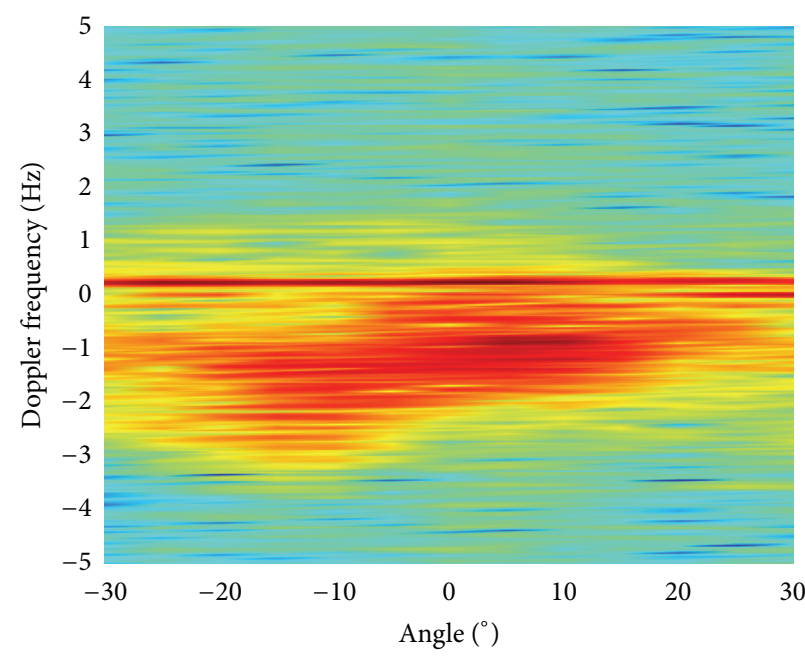

(a) $200 \mathrm{~km}$

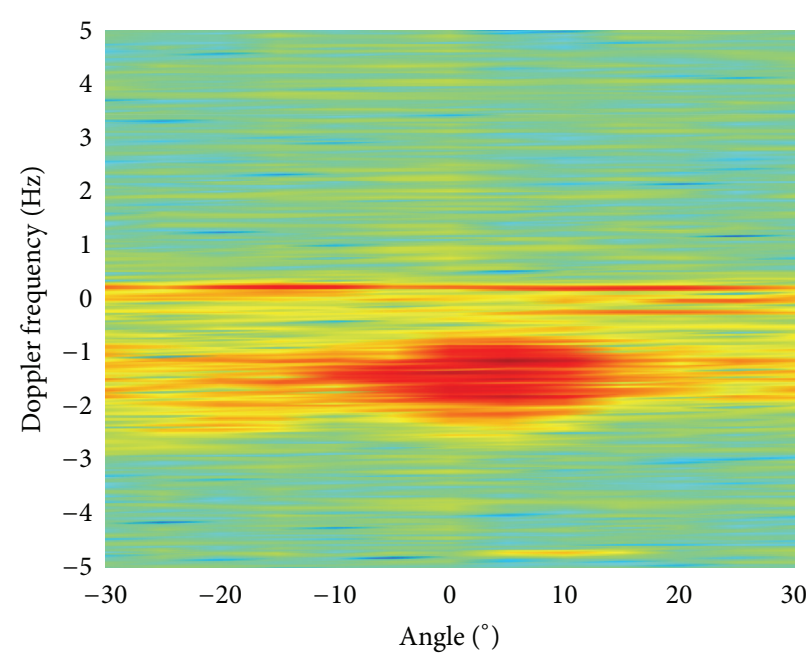

(b) $350 \mathrm{~km}$

Figure 2: Angle-Doppler Map.

2.1. Size of the ADLR. In this part, we try to make an appropriate decision of the size of ADLR in order to make sure that the clutter in this ADLR is as simple as possible. If the data in the ADLR are with high correlation both in beamdomain and frequency-domain, we can consider the clutter in the ADLR simple.

The correlation coefficient of the data with two different beams can be calculated by (1); The correlation coefficient of the data with two different Doppler shifts can be calculated by (2):

$$
\begin{gathered}
\rho_{k, j}=\frac{\left(1 / N_{r}\right) \sum_{r} X_{k}\left(r, f_{d 0}\right) X_{j}^{*}\left(r, f_{d 0}\right)}{\sqrt{\left(1 / N_{r}\right) \sum_{r}\left|X_{k}\left(r, f_{d 0}\right)\right|^{2}\left(1 / N_{r}\right) \sum_{r}\left|X_{j}^{*}\left(r, f_{d 0}\right)\right|^{2}}}, \\
\rho_{f_{d 1}, f_{d 2}}=\frac{\left(1 / N_{r}\right) \sum_{r} X_{f_{d 1}}\left(r, \varphi_{0}\right) X_{f_{d 2}}^{*}\left(r, \varphi_{0}\right)}{\sqrt{\left(1 / N_{r}\right) \sum_{r}\left|X_{f_{d 1}}\left(r, \varphi_{0}\right)\right|^{2}\left(1 / N_{r}\right) \sum_{r}\left|X_{f_{d 2}}^{*}\left(r, \varphi_{0}\right)\right|^{2}}},
\end{gathered}
$$

where $r$ indicates the index of range bins, $N_{r}$ is the number of range bins which are used to calculate the correlation coefficient, $X_{k}$ and $X_{j}$ are the training data with direction $k$ and $j$ with Doppler shift $f_{d 0},(\cdot)^{*}$ is the complex conjugate operator, and $X_{f_{d 1}}$ and $X_{f_{d 2}}$ are the training data with Doppler shift $f_{d 1}$ and $f_{d 2}$ with direction $\varphi_{0}$.

We analyze the angle correlation with different angle intervals as given by (1). The results are shown in Figure 3.

Figure 3(a). The ionospheric clutter exists within the Doppler shifts between $-2 \mathrm{~Hz}$ and $0.12 \mathrm{~Hz}$. The correlation coefficient at beam interval $5^{\circ}$ is greater than the ones when the beam intervals are $10^{\circ}$ and $15^{\circ}$.

Figure 3(b). The correlation coefficient decreases as the beam interval increases. We can deduce from these two figures that the ionospheric clutters have similar characteristics when the beam interval is narrower than $5^{\circ}$.
We also analyze the Doppler frequency correlation with different Doppler shift intervals as given by (2). The results are shown in Figure 4.

Figure 4(a). The ionospheric clutter exists within all the angles ranging from $-30^{\circ}$ to $30^{\circ}$. The correlation coefficient achieves its maximum when the Doppler shift interval is $34 \mathrm{mHz}$. The ones when the Doppler shift intervals are $68 \mathrm{mHz}$ and $102 \mathrm{mHz}$ are almost the same and both less than the one with $34 \mathrm{mHz}$.

Figure 4(b). The correlation coefficient decreases as the Doppler shift interval increases. From these two figures, we can also deduce that the ionospheric clutters have similar characteristics when the Doppler shift interval is less than $34 \mathrm{mHz}$.

In order to maintain the characteristics of ADLR, correlation coefficients within the ADLR data must be high enough. It follows that the size of ADLR cannot be too large. Synthetically, it is preferable to choose the $\pm 5^{\circ}, \pm 34 \mathrm{mHz}$ interval around the cell under test. This is in agreement with the radar theory that correlation coefficient increases as the beam interval and Doppler shift interval decrease.

2.2. Correlation Analysis Method Based on ADJE. In the case of HFSWR, the data in an ADLR cannot be absolutely simple. An ADLR often consists of multiple echo components such as noise, clutter, interference, and targets. The characteristics of ADLRs with different echo components are different. In this part, we mainly analyze the correlation between ADLRs with different echo components.

We first calculate the self-correlation matrix of the data in one ADLR and then obtain the ADJEs through the decomposition of the covariance matrix. In this case, the characteristic of the ADLR is represented by the vector sum of the ADJEs. The following correlation analysis is based on the ADJEs representation.

Firstly, we choose the ADLR which covers $\pm 5^{\circ}$ ( 3 beams) and $\pm 34 \mathrm{mHz}$ (3 Doppler shifts) in one range bin and 


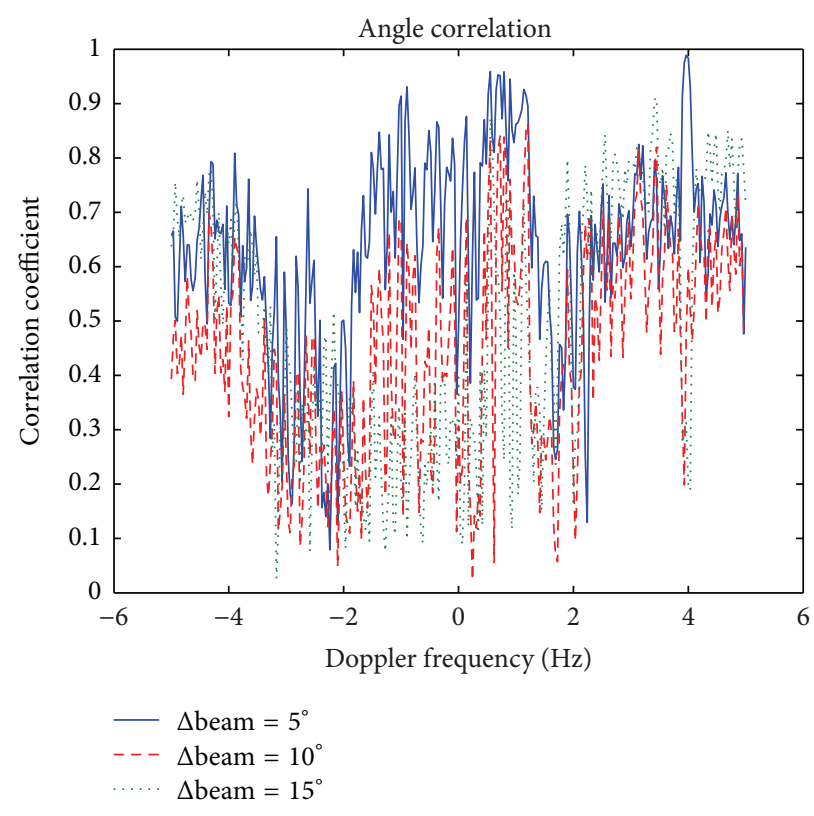

(a) Range $=200 \mathrm{~km}$

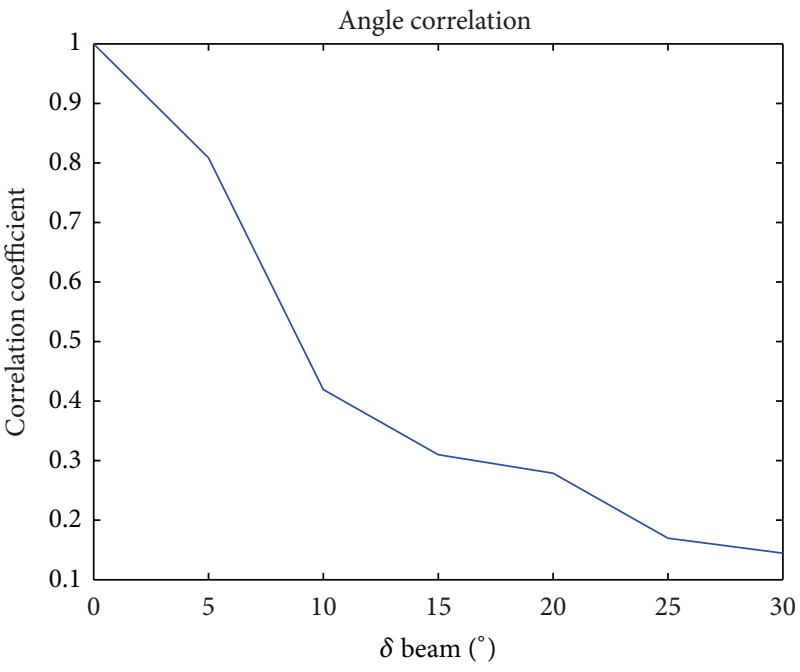

FIgURE 3: Correlation coefficients with different beam intervals.

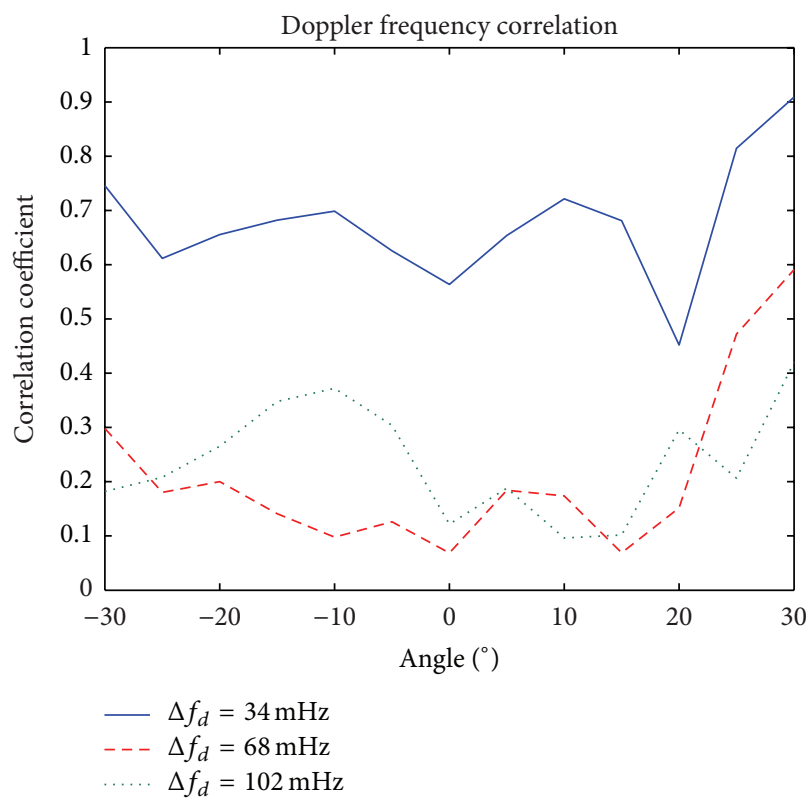

(a) Range $=200 \mathrm{~km}$

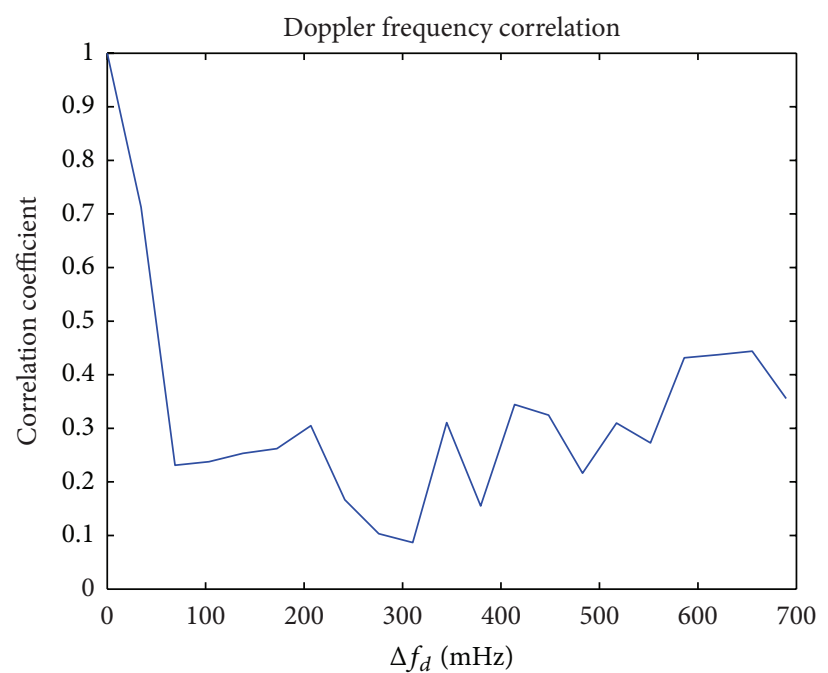

(b) Range $=200 \mathrm{~km}, \varphi_{0}=-10^{\circ}$

FIgURE 4: Correlation coefficients with different Doppler shift intervals.

a $9 \times 1$ vector $\mathbf{X}_{r}$ can be written by Kronecker product. So the self-correlation matrix is $9 \times 9$, and we can get 9 ADJEs by eigen decompostion. A certain number of ADJEs which make greater contribution to represent the characteristics of the ADLR are selected to calculate the correlation coefficient.

The correlation analysis method based on ADJE is as follows.

(i) Calculate the self-correlation matrix $\mathbf{R}_{x}$ of the data $\mathbf{X}_{r}$ in the ADLR with $r$ th range bin by $\mathbf{R}_{x}=\mathbf{X}_{r}^{H} \mathbf{X}_{r}$. (ii) Eigen-decompose $\mathbf{R}_{x}$ to obtain 9 eigenvalues $\lambda_{1}$, $\lambda_{2}, \ldots, \lambda_{9}$ and 9 ADJEs $\xi_{1}, \xi_{2}, \ldots, \xi_{9}$, then the normalized ADJEs by $\bar{\xi}_{i}=\xi_{i} /\left|\xi_{i}\right|, i=1, \ldots, 9$.

(iii) Determine the contribution of the normalized ADJEs $\lambda_{i}\left(\bar{\xi}_{i}, \mathbf{X}_{r}\right), i=1,2, \ldots, 9 ;$

(iv) Choose the normalized ADJE $\boldsymbol{\varsigma}$ which makes the greatest contribution for $\mathbf{X}_{r}$. And $\boldsymbol{\varsigma}$ can represent the characteristic of $\mathbf{X}_{r}$. In the case of HFSWR, especially 
for the Es layer ionospheric clutter, there may be more than one kind of clutter component that exists in an ADLR. Hence, for uncorrelated clutters, there may be two or more dominant normalized ADJEs, and the vector sum of the ADJEs is introduced to represent the characteristics of this ADLR.

(v) Repeat the above procedure; we can obtain $K$ normalized ADJEs $\boldsymbol{\varsigma}_{i}, i=1,2, \ldots, K$ in $K$ different range bins.

(vi) Calculate the correlation coefficients utilizing the $K$ normalized ADJEs $\boldsymbol{\varsigma}_{i}, i=1,2, \ldots, K$ from step (v) by

$$
\rho_{i j}=\boldsymbol{\varsigma}_{i}^{H} \boldsymbol{\varsigma}_{j}, \quad i, j=1,2, \ldots, K .
$$

2.3. SOI Correlation in the Noise Environment. The following analysis is based on the assumption that the noise in HFSWR is Gaussian. We consider two ADLRs: one is with SOI only, and in the other one there exist both SOI and noise, and the signal-to-noise ratio (SNR) is changing.

Gracheva and Cerutti-Maori have analyzed the channel correlation of sea data and have mentioned the relationship between channel correlation and the clutter to noise ratio (CNR) [16]. CNR is defined as

$$
\mathrm{CNR}=\frac{\left(1 / N_{r}\right) \sum_{r}\left\|X_{c}\left(r_{c}\right)\right\|_{2}}{\sigma_{n}^{2}} .
$$

We consider the ADLR in a single range bin $\left(N_{r}=1\right)$. The CNR in this ADLR can be defined as

$$
\mathrm{CNR}_{\mathrm{ADLR}}=\frac{\left\|X_{c}\left(r_{c}\right)\right\|_{2}}{\sigma_{n}^{2}} .
$$

The SNR is defined as

$$
\mathrm{SNR}=\frac{\left\|X_{t}\left(r_{t}\right)\right\|_{2}}{\sigma_{n}^{2}} .
$$

We can replace the $\mathrm{CNR}_{\mathrm{ADLR}}$ in the literature [16] with the SNR as

$$
\rho=\frac{1}{1+1 / \mathrm{SNR}} .
$$

We analyze the correlation between two ADLRs (ADLR and $\mathrm{ADLR}_{2}$ ) by utilizing the algorithm introduced in Section 2.2. The relationship between correlation coefficient and the SNR is obtained via 10000 Monte-Carlo trials.

Under the condition that there are only SOI in $\mathrm{ADLR}_{1}$ and only Gaussian noise in $\mathrm{ADLR}_{2}$, the correlation between $\mathrm{ADLR}_{1}$ and $\mathrm{ADLR}_{2}$ is shown as the red dotted line in Figure 5. The correlation coefficient is kept at a low level. This result is consistent with the radar theory that the SOI is uncorrelated with the noise.

In this case, there are both SOI and noise in $\mathrm{ADLR}_{2}$, and the SNR is variant. The simulation result by (7) is shown as the green dotted line with marker "*” in Figure 5. The correlation coefficient is very close to 1 when the SNR is greater than $20 \mathrm{~dB}$ and very close to zero when the SNR is

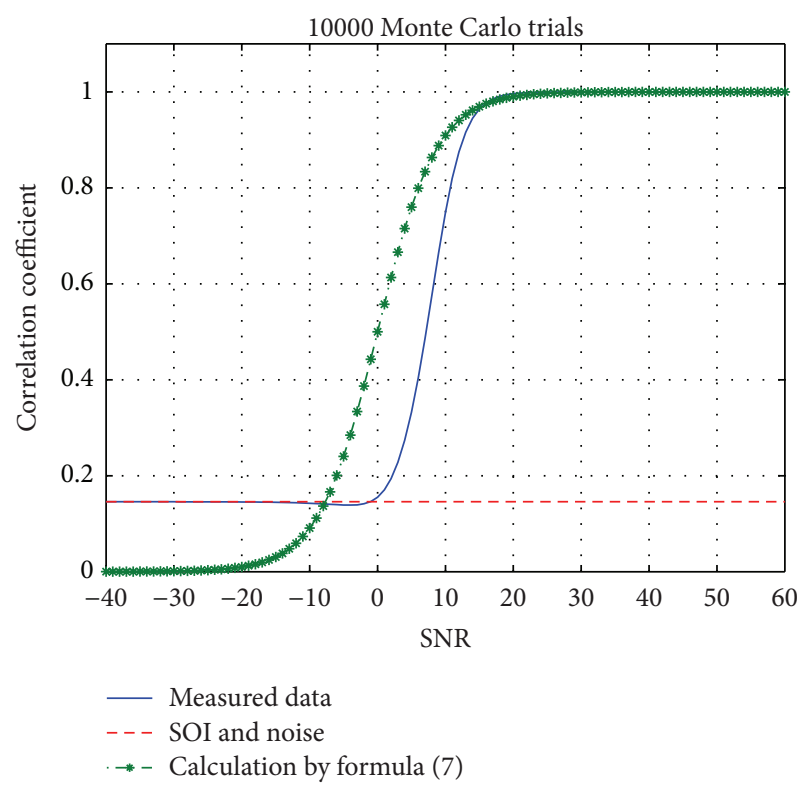

FIGURE 5: The relationship between correlation and SNR.

less than $-20 \mathrm{~dB}$. When $\mathrm{SNR} \in[-20,20] \mathrm{dB}$, the correlation coefficient increases as the SNR increases.

We make a similar analysis with the measured data in $\mathrm{ADLR}_{2}$. The result is shown as a blue solid line in Figure 5. Compared to the simulation results, the correlation coefficient decreases faster as the SNR decreases and the correlation coefficient is greater than zero when the SNR is less than $0 \mathrm{~dB}$. The reason for this error is that the characteristics of SOI and noise could be both reflected in $\mathrm{ADLR}_{2}$ when the SNR is small.

In light of the error present in the above analysis, we modify the equation as follows.

We mark the $\mathrm{ADLR}_{1}$ which only contains $\mathrm{SOI}_{1}$ as $\mathrm{SD}_{1}$ and the Doppler-angle data as $\mathbf{Z}_{t}$; we also mark the $\mathrm{ADLR}_{2}$ which contains $\mathrm{SOI}_{2}$ and Gaussian noise as $\mathrm{SD}_{2}$ and the Doppler-angle data as $\mathbf{Z}_{s n}$, and the power of the noise is $\sigma^{2}$. We calculate the normalized ADJEs of ADLR 1 and $\mathrm{ADLR}_{2}$ utilizing $\mathbf{Z}_{t}$ and $\mathbf{Z}_{s n}$ for $\boldsymbol{\xi}_{t}$ and $\boldsymbol{\xi}_{s n}$, respectively. So $\boldsymbol{\xi}_{s n}$ can be written as

$$
\xi_{s n} \approx \frac{\lambda_{t} \xi_{t}^{\prime}+\sigma^{2} \xi_{n}}{\sqrt{\lambda_{t}^{2}+\sigma^{4}}}
$$

where $\lambda_{t}$ is the eigenvalue of $\mathrm{SOI}_{2}, \xi_{t}^{\prime}$ is the eigenvector of $\mathrm{SOI}_{2}$, and $\xi_{n}$ is the eigenvector of noise.

So the correlation coefficient can be written as

$$
\begin{aligned}
\rho & =\boldsymbol{\xi}_{t}^{H} \boldsymbol{\xi}_{s n} \\
& \approx \xi_{t}^{H} \cdot \frac{\lambda_{t} \boldsymbol{\xi}_{t}^{\prime}+\sigma^{2} \boldsymbol{\xi}_{n}}{\sqrt{\lambda_{t}^{2}+\sigma^{4}}} \\
& =\frac{\lambda_{t}}{\sqrt{\lambda_{t}^{2}+\sigma^{4}}} \cdot \frac{1}{1+1 / \mathrm{SNR}}+\frac{\sigma^{2}}{\sqrt{\lambda_{t}^{2}+\sigma^{4}}} \cdot \rho_{s n},
\end{aligned}
$$


where $\xi_{t}^{H} \xi_{t}^{\prime}=1 /(1+1 / \mathrm{SNR}), \rho_{s n}$ is a statistical value related to the SOI and distribution of the noise, $\rho_{s n}=\xi_{t}^{H} \xi_{n}$.

When $\mathrm{SOI}_{2}$ is dominant in $\mathrm{SD}_{2}, \boldsymbol{\xi}_{s n} \approx \boldsymbol{\xi}_{t}^{\prime}$, the correlation coefficient is $\rho=\boldsymbol{\xi}_{t}^{H} \boldsymbol{\xi}_{t}^{\prime}=1 /(1+1 / \mathrm{SNR})$.

When noise is dominant in $\mathrm{SD}_{2}, \xi_{s n} \approx \xi_{n}$, correlation coefficient is $\rho=\boldsymbol{\xi}_{t}^{H} \boldsymbol{\xi}_{n}=\rho_{s n}$.

The simulation result based on (9) are shown as a black dot-dash line in Figure 6 fits the measured data much better than the simulation result given by (7) does.

2.4. SOI Correlation in the Ionospheric Clutter Environment. We mark the $\mathrm{ADLR}_{1}$ which only contains $\mathrm{SOI}_{1}$ as $\mathrm{SD}_{1}$ and the Doppler-angle data as $\mathbf{Z}_{t}\left(r_{t}\right)$; we also mark the $\mathrm{ADLR}_{3}$ which contains $\mathrm{SOI}_{2}$ and the ionospheric clutter as $\mathrm{SD}_{3}$ and the Doppler-angle data as $\mathbf{Z}_{c}\left(r_{c}\right)$, and $r_{t}$ and $r_{c}$ are the range bins of $\mathrm{SD}_{1}$ and $\mathrm{SD}_{3}$. The SCR of $\mathrm{SOI}_{2}$ and the ionospheric clutter are written as

$$
\operatorname{SCR}=\frac{\left\|\mathbf{Z}_{t}\left(r_{t}\right)\right\|_{2}}{\left(1 / N_{r}\right) \sum_{r}\left\|\mathbf{Z}_{c}\left(r_{c}\right)\right\|_{2}} .
$$

We analyze the SOI correlation in the ionospheric clutter background as in Section 2.3. We can get the following:

$$
\rho= \begin{cases}\frac{1}{1+1 / \mathrm{SCR}}, & \text { for SCR }>H_{\mathrm{SCR}}, \\ \rho_{s c}, & \text { for SCR }<L_{\mathrm{SCR}} \\ \frac{\lambda_{t}}{\sqrt{\lambda_{t}^{2}+\lambda_{c}^{2}} \cdot \frac{1}{1+1 / \mathrm{SCR}}} & \\ +\frac{\lambda_{c}}{\sqrt{\lambda_{t}^{2}+\lambda_{c}^{2}}} \cdot \rho_{s c}, & \text { others, }\end{cases}
$$

where $\rho_{s c}$ is the correlation coefficient between SOI and the ionospheric clutter, $\lambda_{t}$ and $\lambda_{c}$ are the eigenvalues of $\mathrm{SOI}_{2}$ and the ionospheric clutter in $\mathrm{ADLR}_{3}$, and $L_{\mathrm{SCR}}$ and $H_{\mathrm{SCR}}$ are the upper limit and lower limit of SCR, respectively, and they are related to the characteristics of the ionospheric clutter.

The correlation of SOI in the ionospheric clutter environment utilizing the measured data at the range of $180 \mathrm{~km}$ and $185 \mathrm{~km}$ is shown in Figure 7.

If there is only ionospheric clutter in $\mathrm{ADLR}_{3}$, the correlation coefficients between $\mathrm{ADLR}_{1}$ and $\mathrm{ADLR}_{2}$ are shown as a red-dotted line. It is related to the characteristic of ionospheric clutter and has different values at different ranges. The result of correlation analysis utilizing measured data is shown as a blue solid line. And the result calculated by (11) fits the measured data well.

It can be seen from (11) and Figure 7 that the relationship between correlation and SCR can be divided into three parts:

(a) the correlation coefficient keeps a low value when the SCR is small, and it mainly depends on the characteristics of the ionospheric clutter;

(b) when the SCR is large enough, the correlation coefficient is close to 1 ;

(c) when the SCR is an intermediate value, the correlation coefficient is related not only to the SCR but also to the characteristics of SOI and ionospheric clutter.

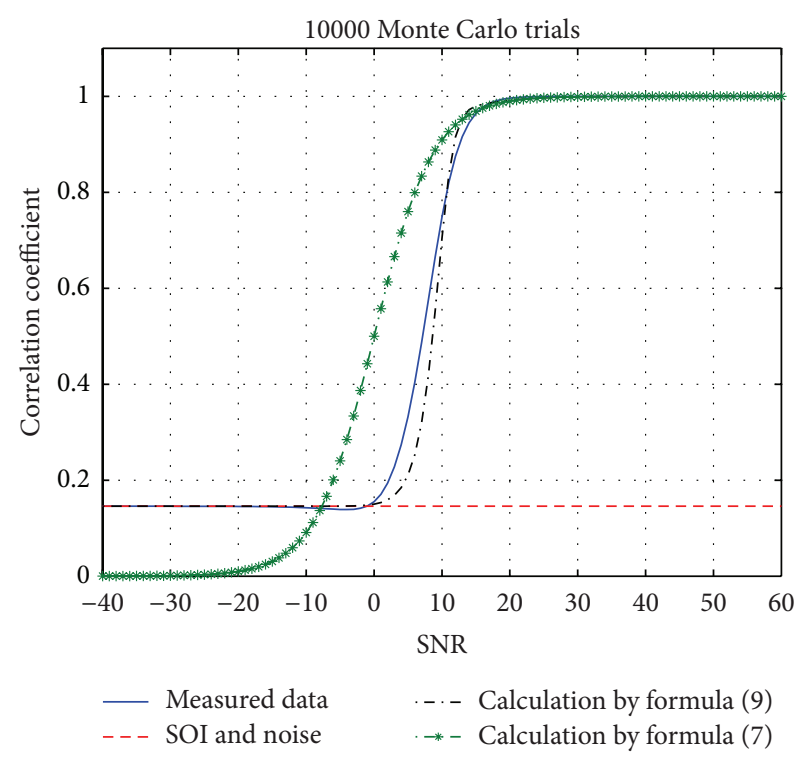

FIGURE 6: The relationship between correlation and SNR.

2.5. Range Correlation of Nonhomogeneous Ionospheric Clutter. The motion of ionosphere is complex with the major factor of nonuniform plasma. Due to this complex motion, the ionospheric clutter in HFSWR is nonhomogeneous.

We calculate the correlation coefficients of ADLRs in different ranges within $150 \mathrm{~km}$ to $430 \mathrm{~km}$ referred to $225 \mathrm{~km}$ at the Doppler shift $-1.2 \mathrm{~Hz}$ utilizing the algorithm mentioned in Section 2.2. The result is shown in Figure 8. The correlation of nonhomogeneous ionospheric clutter decreased as the range interval increased. But there may be a few high correlated range data due to the nonhomogeneous characteristics of the ionospheric clutter.

\section{Strategy of Choosing the Training Data Based on Correlation}

The full STAP algorithm is ideal and requires the training data to meet two conditions. One is that the clutter must be independent, and identically distributed. The other is that the number of the training data must be twice greater than the degrees of freedom of the clutter [17].

In HFSWR, the first condition is difficult to meet due to the complex echoes. The long coherent integrated time (CIT) leads to the large degrees of freedom. So it requires considerable training data which are difficult to be attained in the practical system. Compared with the fully STAP, the partial STAP algorithm has the advantage of less computation. So the partial STAP has become the researchers priority.

The JDL algorithm is one of the partial STAP algorithms. It can reduce the degrees of freedom by using a transformation matrix $\mathbf{T}$. Therefore, it can solve the problem of the limited training data and it also can reduce the computation.

The result of Section 2.5 showed that the range of correlation of the nonhomogeneous ionospheric clutter rise, and fall irregularly. In order to suppress the nonhomogeneous ionospheric clutter effectively utilizing the JDL, we should 


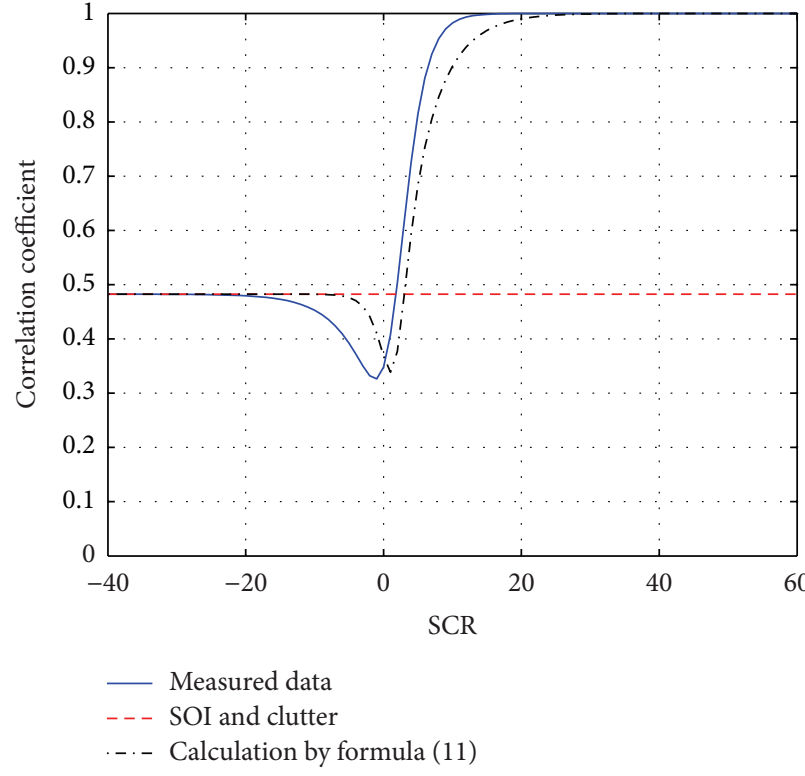

(a) Range $=180.00 \mathrm{~km}$

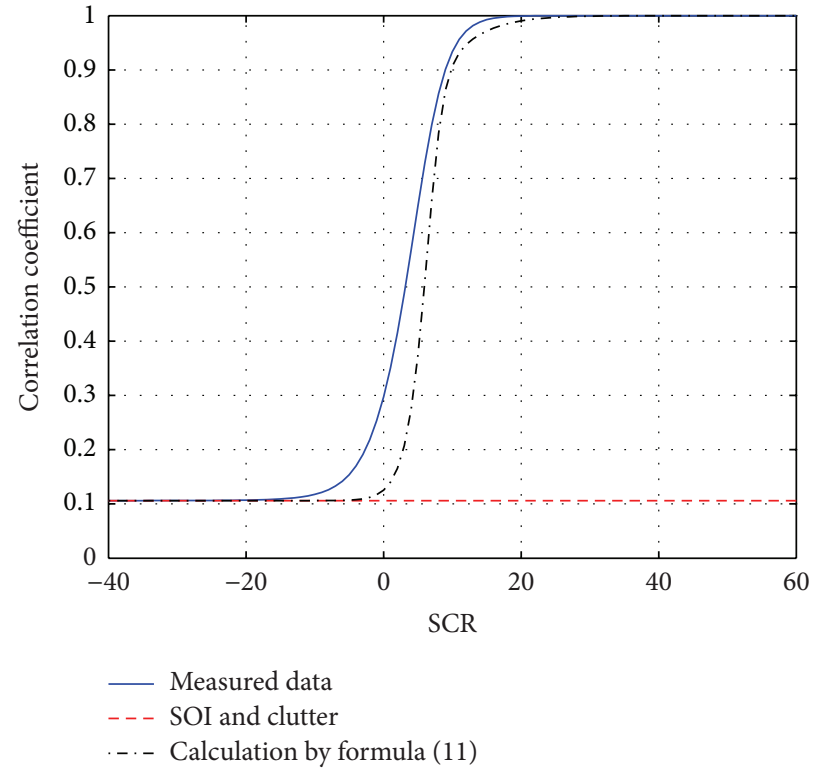

(b) Range $=185.00 \mathrm{~km}$

FIGURE 7: The relationship between correlation and SCR.

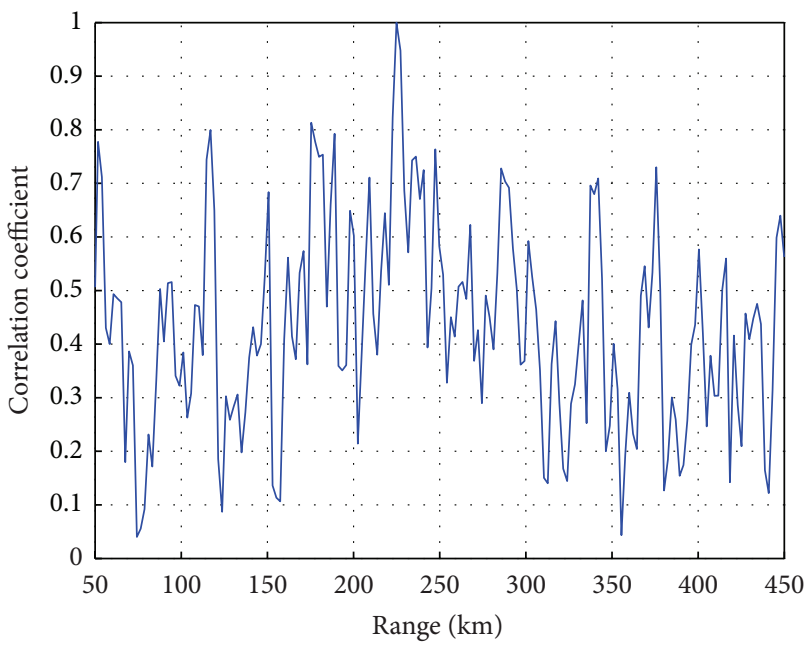

FIGURE 8: Range correlation of ionospheric clutter at $-1.2 \mathrm{~Hz}$.

ensure that the training data are highly related with the clutter cell under test.

3.1. Strategy Based on the Correlation. In this part, we choose the appropriate samples as the training data to obtain the covariance matrix more accurately based on the correlation analysis above.

The receiver antenna array of HFSWR is described as the $N$ elements uniform linear array (ULA). $d$ is the interelement spacing, a coherent integrated time contains $M$ pulses, $T$ is the pulse repetition time, and $f_{s}$ is the system sampling rate. For a given range bin, the data sampled by the $N$ elements form an $\mathrm{N}$-dimensional vector, and the data received by one element in a CIT form an $M$-dimensional vector. So that an $N M \times 1$ dimensional vector of the space-time snapshots in $l$ th range bin is formed as defined by

$$
\mathbf{X}_{l}=\left[\begin{array}{llll}
\mathbf{x}_{1} & \mathbf{x}_{2} & \cdots & \mathbf{x}_{M}
\end{array}\right]^{T},
$$

where $\mathbf{x}_{i}=\left[\begin{array}{llll}x_{i, 1} & x_{i, 2} & \cdots & x_{i, N}\end{array}\right]^{T}, i=1,2, \ldots, M$.

We define the space-time steering vector as

$$
\mathbf{v}\left(\phi_{t}, f_{t}\right)=\mathbf{b}\left(f_{t}\right) \otimes \mathbf{a}\left(\phi_{t}\right),
$$

where $\mathbf{a}\left(\phi_{t}\right)$ is a space steering vector as defined by (14) and $\mathbf{b}\left(f_{t}\right)$ is a time steering vector as defined by (15):

$$
\begin{aligned}
& \mathbf{a}\left(\phi_{t}\right)=\left[\begin{array}{lllll}
1 & Z_{s} & Z_{s}^{2} & \cdots & Z_{s}^{N-1}
\end{array}\right]^{T}, \\
& \mathbf{b}\left(f_{t}\right)=\left[\begin{array}{lllll}
1 & Z_{t} & Z_{t}^{2} & \cdots & Z_{t}^{M-1}
\end{array}\right]^{T},
\end{aligned}
$$

where $Z_{s}=e^{j 2 \pi(d / \lambda) \sin \phi_{t}}$ and $Z_{t}=e^{j 2 \pi\left(f_{t} / f_{R}\right)}$.

JDL algorithm can transform the independent range samples to the LPR by using the transformation matrix $\mathbf{T}$ to reduce the degrees of freedom. Thus, $\mathrm{T}$ is the key of the JDL algorithm. Wang has discussed how to choose the LPR in the literature [18]: the clutter can be suppressed more effectively as the LPR larger, at the same time the heavily computational cost is a big problem. With all things considered in the case of HFSWR, the LPR we are concerned about contains three angle units and three Doppler shift units $\left(\eta_{a}=3, \eta_{d}=\right.$ 3 ), which is the same size as the ADLR we mentioned in Section 2. So that the transformation matrix $\mathbf{T}$ can be written as

$$
\mathbf{T}=\left[\begin{array}{lll}
\mathbf{b}\left(f_{-1}\right) & \mathbf{b}\left(f_{0}\right) & \mathbf{b}\left(f_{1}\right)
\end{array}\right] \otimes\left[\begin{array}{lll}
\mathbf{a}\left(\phi_{-1}\right) & \mathbf{a}\left(\phi_{0}\right) & \mathbf{a}\left(\phi_{1}\right)
\end{array}\right] .
$$




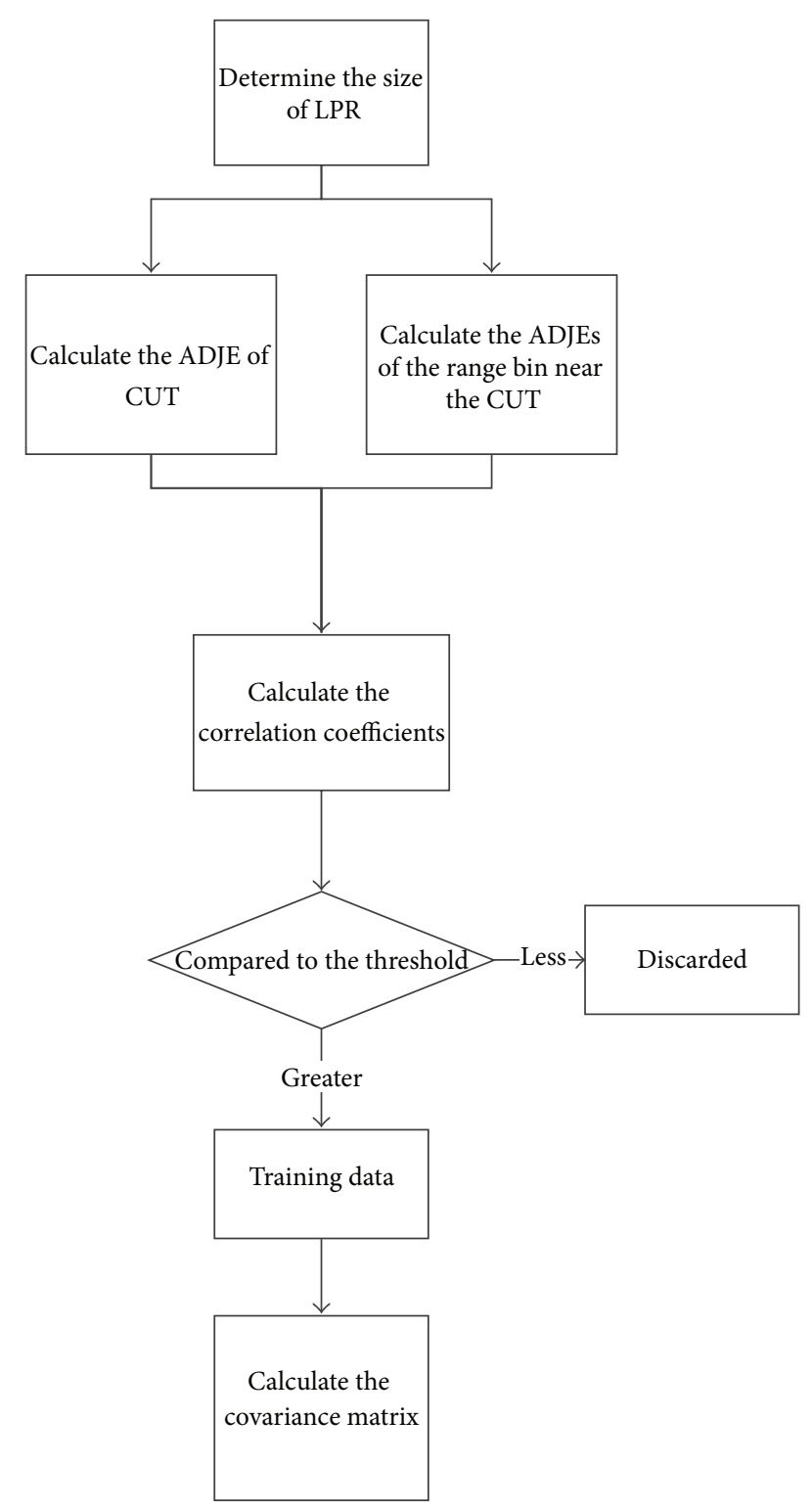

FIGURE 9: Implementation of improved-JDL.

Thus the new range samples and the new space-time steering vector in JDL can be written as

$$
\widetilde{\mathbf{X}}_{l}=\mathbf{T}^{H} \cdot \mathbf{X}_{l}, \quad \widetilde{\mathbf{v}}=\mathbf{T}^{H} \cdot \mathbf{v} .
$$

Then, we analyze the correlation of the range samples utilizing algorithm in Section 2. We choose the $K$ training data which is highly correlated with the cell under test (CUT) by setting a threshold. Finally, we calculate the covariance matrix $\widehat{\mathbf{R}}$ utilizing the $K$ chosen training data as defined by

$$
\widehat{\mathbf{R}}=\frac{1}{K} \sum_{0}^{K-1} \mathbf{Z}_{k} \mathbf{Z}_{k}^{H} .
$$

We assume that CUT is the $t$ th range bin. $\mathbf{X}_{t}$ is the sample in CUT. Therefore, training data $\mathbf{Z}_{k}$ should be highly correlated with the sample $\mathbf{X}_{t}$. And $\mathbf{Z}_{k}$ are chosen from
$\mathbf{X}_{l}, l=1,2, \ldots, t-p, t+p, \ldots$ where $p$ is the number of protected range bins which are used to keep the training data to be independent of $\mathbf{X}_{t}$. That is because the target in HFSWR often spreads a few range bins, and the range samples in these range bins are nonindependent.

When we get the covariance matrix $\widehat{\mathbf{R}}$, the optimal weights can be expressed as

$$
\mathbf{W}_{\text {opt }}=\widehat{\mathbf{R}}^{-1} \widetilde{\mathbf{v}}
$$

The implementation of the improved-JDL can be shown as in the following steps and Figure 9.

(i) Determine the size of LPR and the transformation matrix $\mathbf{T}$ as shown in (16).

(ii) Calculate the ADJEs of CUT and the range samples near the CUT as mentioned in steps (i) (v) in Section 2.2

(iii) Calculate the correlation coefficients between the CUT and the nearby range samples as (3).

(iv) Compare the correlation coefficients with the threshold $c_{0}$. If it is greater than $c_{0}$, these range samples can be treated as training data; otherwise it will be discarded.

(v) Calculate the covariance matrix of the ionospheric clutter utilizing the chosen training data as (18).

3.2. Threshold Chosen. The key point of the improved-JDL is the threshold $c_{0}$. Due to different $c_{0}$, the chosen training data can be absolutely different. This can result in different covariance matrix and space-time weights. Curve of the improvement factor (IF) and the threshold is shown in Figure 10. The IF is no longer improved as the threshold raised when the threshold is greater than 0.4. Thus, we can choose the threshold that is greater than 0.4 for single target situation.

In the case of HFSWR, the targets often travel with batch. In this condition, the interference between targets must be concerned. Figure 11 shows the correlation between the range sample 1 with only strong target and the range sample 2 with weak target and the ionospheric clutter. The SCR in Figure 11 is the weak target to ionospheric clutter ratio in range sample 2.

When the SCR $<0 \mathrm{~dB}$, the correlation coefficients between the strong target and the weak target are mostly less than 0.8 . When the SCR $<-10 \mathrm{~dB}$, the correlation coefficients are mostly less than 0.7. As the threshold increased, the number of chosen training data may decrease and the covariance matrix may be calculated inexactly because there are not enough training data.

With both of the correlation and the number of training data considered, the threshold should be little greater than 0.7. Thus it cannot only keep the training meeting highly correlated but also avoid the interferences of the strong targets.

\section{Results of Measured Data}

We inject one target in the measured data represented in Figure 1 for the single target situation and two targets for 


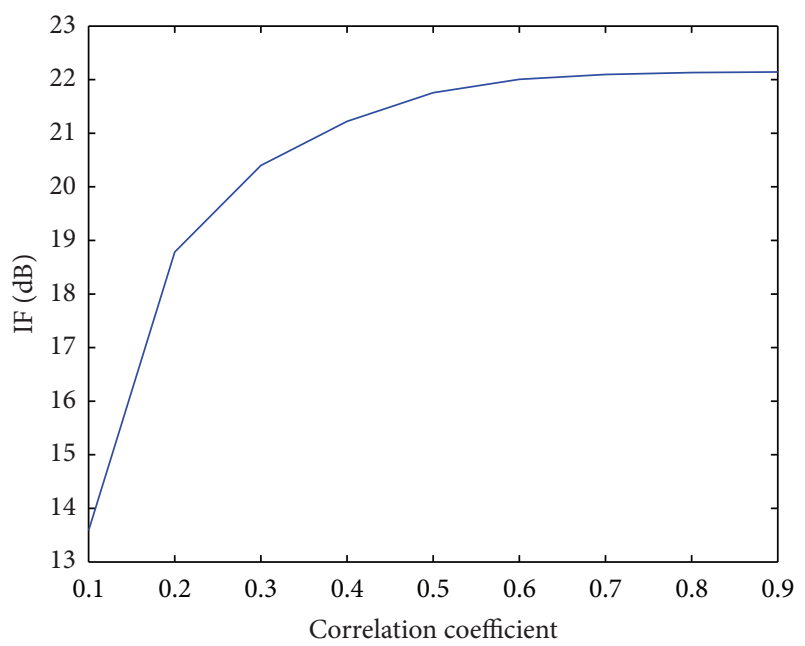

FIGURE 10: The relationship between the IF and correlation coefficient threshold.

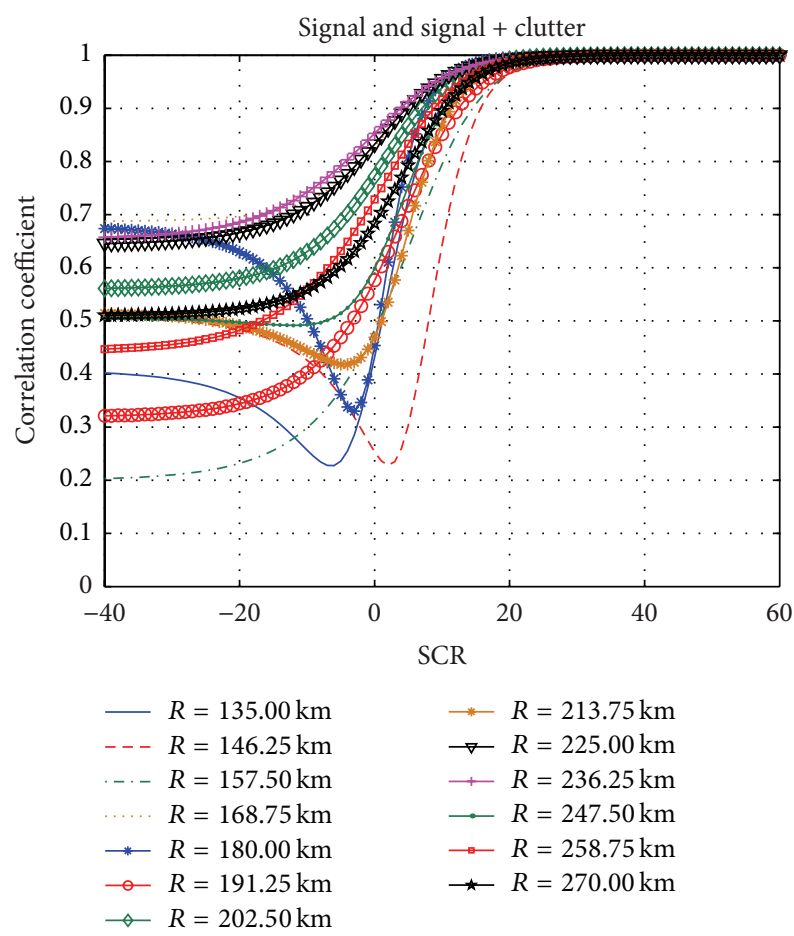

FIGURE 11: Correlation between the strong target and the weak target within ionospheric clutter.

TABLE 1: Single target situation.

\begin{tabular}{lccc}
\hline Range $(\mathrm{km})$ & Doppler shift $(\mathrm{Hz})$ & Angle $\left(^{\circ}\right)$ & SCR $(\mathrm{dB})$ \\
\hline 200.25 & -1.2 & 0 & 5 \\
\hline
\end{tabular}

the multitargets situation. The parameters of the targets are shown in Tables 1 and 2.

For the single target situation, the injected target can be detected when the threshold is over 0.6 as shown in Figure 12. Compared with the results of the three thresholds, the IF is
TABLE 2: Mutitargets situation.

\begin{tabular}{lccc}
\hline Range $(\mathrm{km})$ & Doppler shift $(\mathrm{Hz})$ & Angle $\left(^{\circ}\right)$ & SCR $(\mathrm{dB})$ \\
\hline 200.25 & -1.2 & 0 & 0 \\
211.25 & -1.2 & 0 & 10 \\
\hline
\end{tabular}

TABLE 3: Range-spread targets situation.

\begin{tabular}{lccc}
\hline Range $(\mathrm{km})$ & Doppler shift $(\mathrm{Hz})$ & Angle $\left(^{\circ}\right)$ & SCR $(\mathrm{dB})$ \\
\hline $186.75-191.25$ & -1.2 & 0 & 0 \\
$227.25-231.75$ & -1.2 & 0 & 10 \\
\hline
\end{tabular}

almost the same. That is in agreement with the conclusion in Section 3.2. However, there are four fake targets at $159.75 \mathrm{~km}$, $231.75 \mathrm{~km}, 265.5 \mathrm{~km}$, and $319.5 \mathrm{~km}$ when the threshold is 0.8 . And two fake targets exist when the threshold is 0.7. When the threshold decreases to 0.6 , there is no fake target any more. That is because when we process the data in these ranges with threshold 0.7 or 0.8 , the training data we have obtained is too few to suppress the ionospheric clutter when the threshold is higher. In this case, the energy of the ionospheric clutter stays at a high level and it is easy to be treated as a "fake target." But the number of the training data increased as the threshold decreased. Thus, there is no fake target when the threshold is lower.

So for the single target situation, the threshold is set mainly considering the fake targets.

For the multitargets' situation, the results are shown in Figure 13. Focusing on the two-injected targets at range of $200.25 \mathrm{~km}$ and $211.25 \mathrm{~km}$, they can both be detected when the threshold is 0.7 or 0.8 . And the IF of target 1 is almost the same. But when the threshold is 0.6 , the energy of target 1 is weakened. That is because is when the threshold down to 0.6 , target 2 is treated as training data. Thus, the covariance matrix of the ionospheric clutter contains the characteristics of target, and so target 1 is weakened.

Similar to the single target situation, there may be fake targets when the thresholds are high and the number of fake targets decreased as the threshold increased. In the actual situation, the multitargets situation is common. So the analysis of this part can make great sense.

Considering both the IF and fake targets problem, the threshold should be set within 0.6 and 0.7 when the improved-JDL algorithm is utilized in HFSWR.

We also consider the range-spread targets in HFSWR as shown in Table 3; we can suppress the ionospheric clutter and detect the two targets as shown in Figure 14 utilizing the improved-JDL method with correlation threshold $c_{0}=0.6$ and the number of protected range bins $p=2$.

\section{Conclusion}

To counter the nonhomogeneous ionospheric clutter background of HFSWR, this paper proposes a feature analytical algorithm based on the Angle-Doppler Joint Eigenvector to analyze the range correlation of the nonhomogeneous ionospheric clutter. It turns out that the range correlation coefficient is irregular in its variation. In light of this prior 


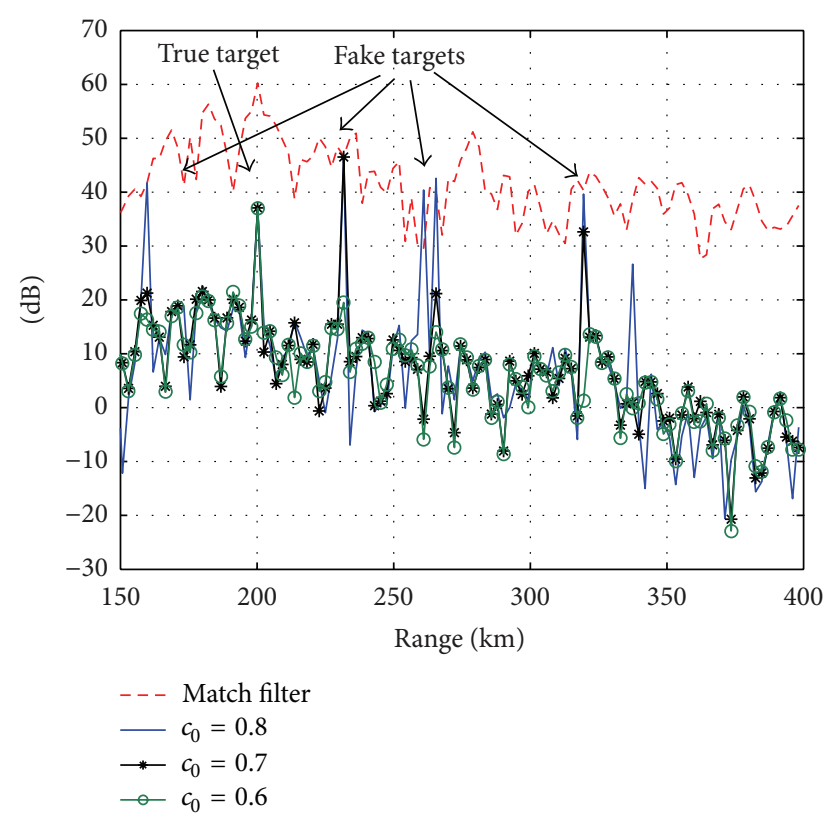

FIGURE 12: The results by using the improved-JDL for single target situation.

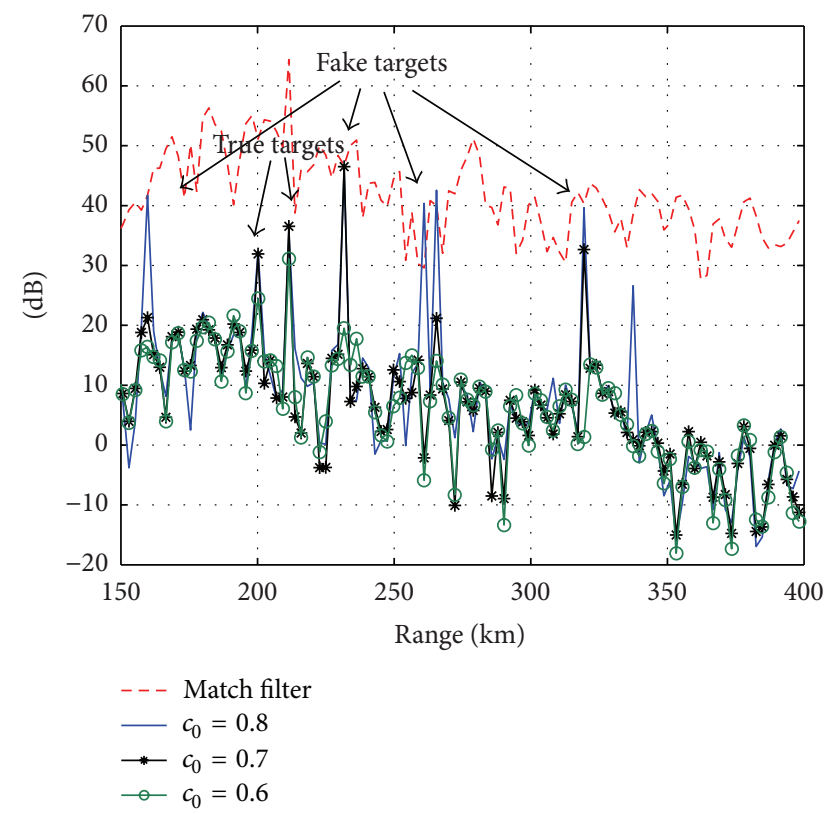

FIGURE 13: The results by using the improved-JDL for multitarget situation.

knowledge, a further step is taken to deal with the weak target detection problem in HFSWR by analyzing the correlation between targets' signals and the ionospheric clutter and the negative effect imposed by strong targets on weak targets detection. To sum up, this paper proposes a correlation based training data chosen strategy for the JDL algorithm, and discusses the corresponding decision threshold selection in detail. Consequently, decision thresholds should be set up according to the practical situation of the nonhomogeneous

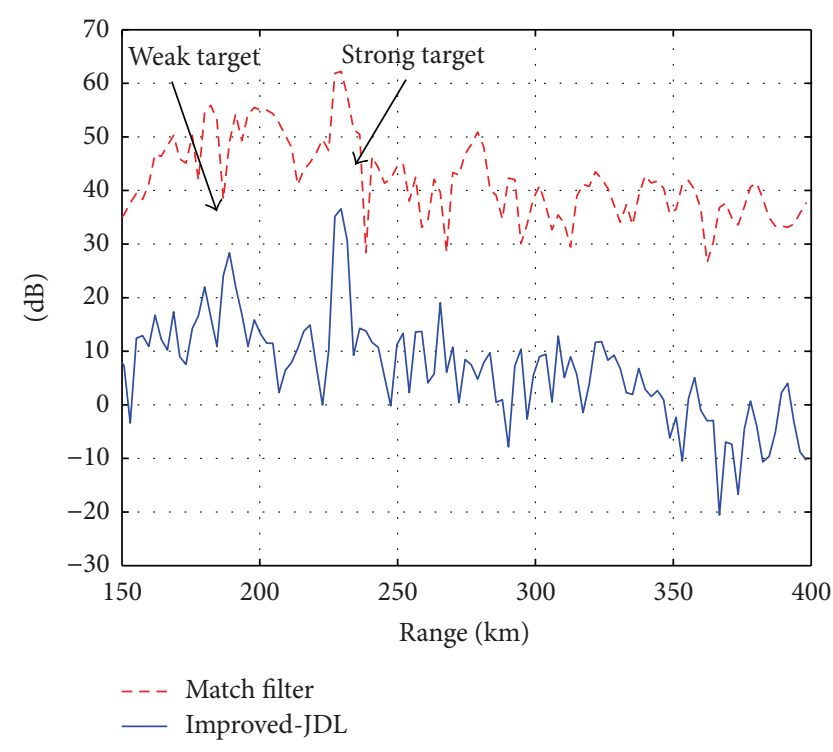

FIgURE 14: The results by using the improved-JDL for multitarget situation (range-spread targets).

ionospheric clutter and values between 0.6 and 0.7 are preferable. This improved-JDL algorithm is validated by measured data which shows that the weak target detection performance can be notably improved in the background of nonhomogeneous ionospheric clutter.

\section{Acknowledgment}

The authors express gratitude to the Institute of Electronic and Information Technology, which provided the HFSWR data, and to those who helped during the writing of this paper. This work was supported in part by the National Science Fund Committee (NSFC) under Grant 61032011.

\section{References}

[1] T. Mao, W. Xia, C. Qu, and J. Luo, "A study on characteristics and applications of HF ground wave OTH radar," Modern Radar, no. 3, pp. 7-10, 2009.

[2] L. E. Brennan and L. S. Reed, "Theory of adaptive radar," IEEE Transactions on Aerospace and Electronic Systems, vol. 9, no. 2, pp. 237-252, 1973.

[3] S. Bidon, O. Besson, and J.-Y. Tourneret, "Knowledge-aided STAP in heterogeneous clutter using a hierarchical bayesian algorithm," IEEE Transactions on Aerospace and Electronic Systems, vol. 47, no. 3, pp. 1863-1879, 2011.

[4] X. Zhu, J. Li, and P. Stoica, "Knowledge-aided space-time adaptive processing," IEEE Transactions on Aerospace and Electronic Systems, vol. 47, no. 2, pp. 1325-1336, 2011.

[5] J. Wu, T. Wang, X. Meng, and Z. Bao, "Clutter suppression for airborne non-sidelooking radar using ERCB-STAP algorithm," IET Radar, Sonar and Navigation, vol. 4, no. 4, pp. 497-506, 2010.

[6] Y. R. Zheng, T. Shao, and E. Blasch, "A fast-converging spacetime adaptive processing algorithm for non-Gaussian clutter suppression," Digital Signal Processing, vol. 22, no. 1, pp. 74-86, 2012. 
[7] W. L. Melvin, "A STAP overview," IEEE Aerospace and Electronic Systems Magazine, vol. 19, no. 1, pp. 19-35, 2004.

[8] F. Letestu, "Space-time adaptive processing for navigation," in Proceedings of the International Radar Symposium (IRS '11), pp. 769-773, September 2011.

[9] J. Xie, Y. Yuan, and Y. Liu, "Suppression of sea clutter with orthogonal weighting for target detection in shipborne HFSWR," IEE Proceedings: Radar, Sonar and Navigation, vol. 149, no. 1, pp. 39-44, 2002.

[10] G. Fabrizio, D. Holdsworth, and A. Farina, "Experimental HF radar trial of real-time STAP," in Proceedings of the International Conference on Waveform Diversity and Design (WDD '07), pp. 316-320, June 2007.

[11] R. S. Adve, T. B. Hale, and M. C. Wicks, "Practical joint domain localised adaptive processing in homogeneous and nonhomogeneous environments-part 1: homogeneous environments," IEE Proceedings: Radar, Sonar and Navigation, vol. 147, pp. 5765, 2000.

[12] R. S. Adve, T. B. Hale, and M. C. Wicks, "Practical joint domain localised adaptive processing in homogeneous and nonhomogeneous environments-part 2: nonhomogeneous environments," IEE Proceedings: Radar, Sonar and Navigation, vol. 147, pp. 66-74, 2000.

[13] M. Ravan, O. Saleh, and R. S. Adve, "KB-STAP Implementation for HFSWR," Final Reprot, University of Toronto, 2008.

[14] O. Saleh, R. S. Adve, and R. J. Riddolls, "Fast fully adaptive processing: a multistage STAP approach," in Proceedings of the IEEE Radar Conference (RADAR '09), pp. 1-6, May 2009.

[15] H. Wang and L. Cai, "On adaptive spatial-temporal processing for airborne surveillance radar systems," IEEE Transactions on Aerospace and Electronic Systems, vol. 30, no. 3, pp. 660-670, 1994.

[16] V. Gracheva and D. Cerutti-Maori, "Multi-channel analysis of sea clutter for STAP applications," in Proceedings of the 9th European Conference on Synthetic Aperture Radar (EUSAR '12), pp. 195-198, 2012.

[17] I. S. Reed, J. D. Mallett, and L. E. Brennan, "Rapid convergence rate in adaptive arrays," IEEE Transactions on Aerospace and Electronic Systems, vol. 10, no. 6, pp. 853-863, 1974.

[18] Z. Wang, Research on STAP-based ionospheric clutter suppressing method in high frequency surface wave radar [M.S. thesis], Harbin Institute of Technology, Harbin, China, 2010. 

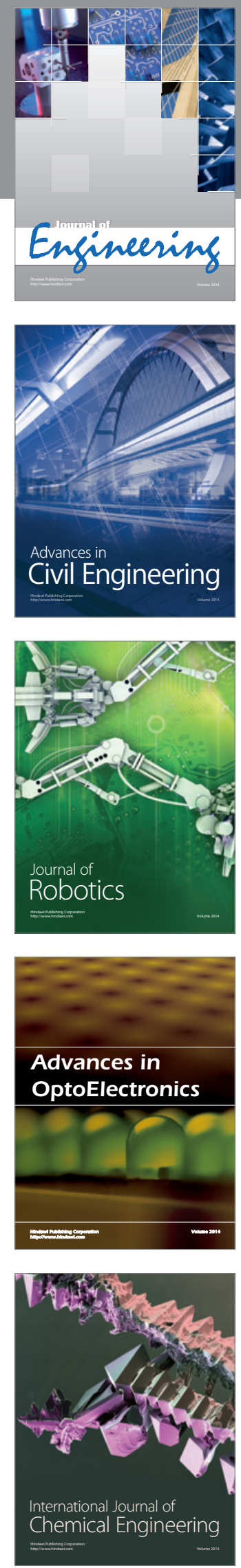

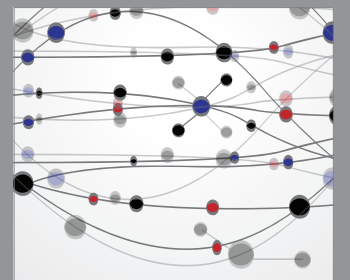

The Scientific World Journal
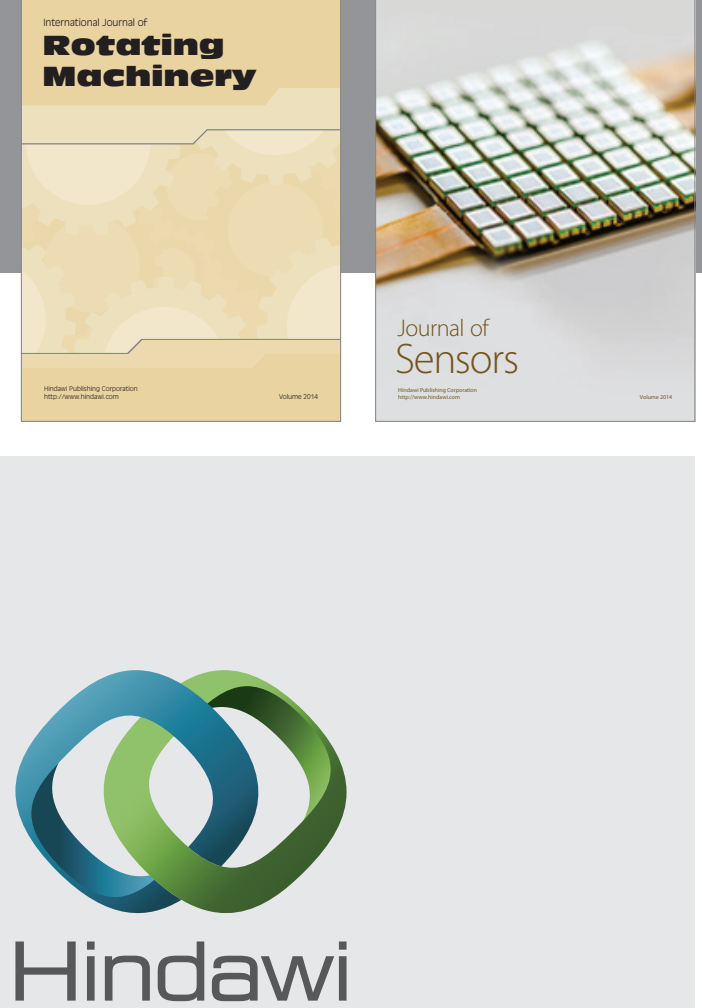

Submit your manuscripts at http://www.hindawi.com
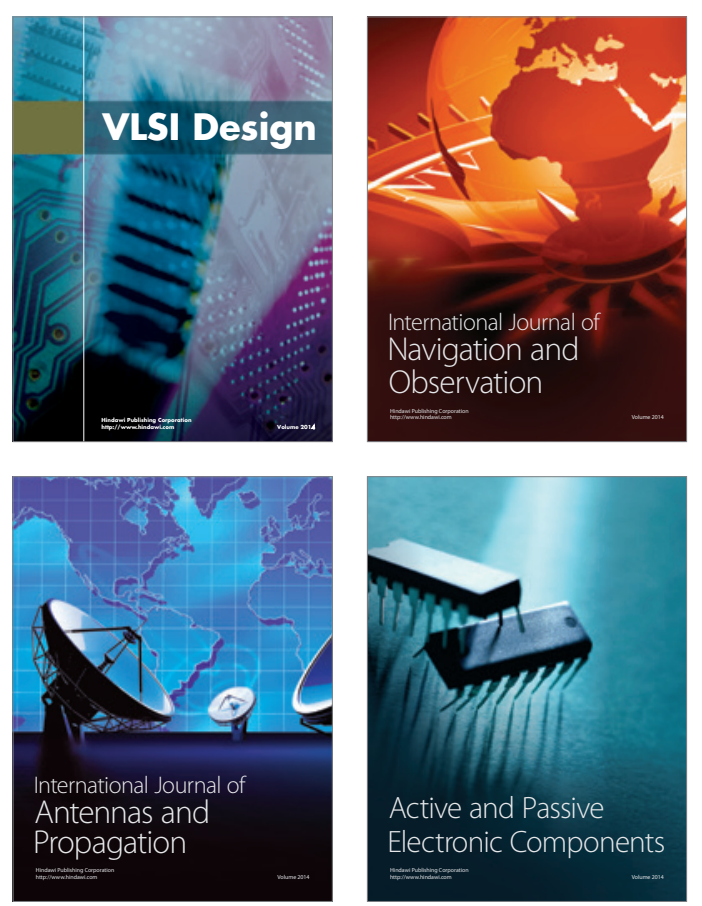
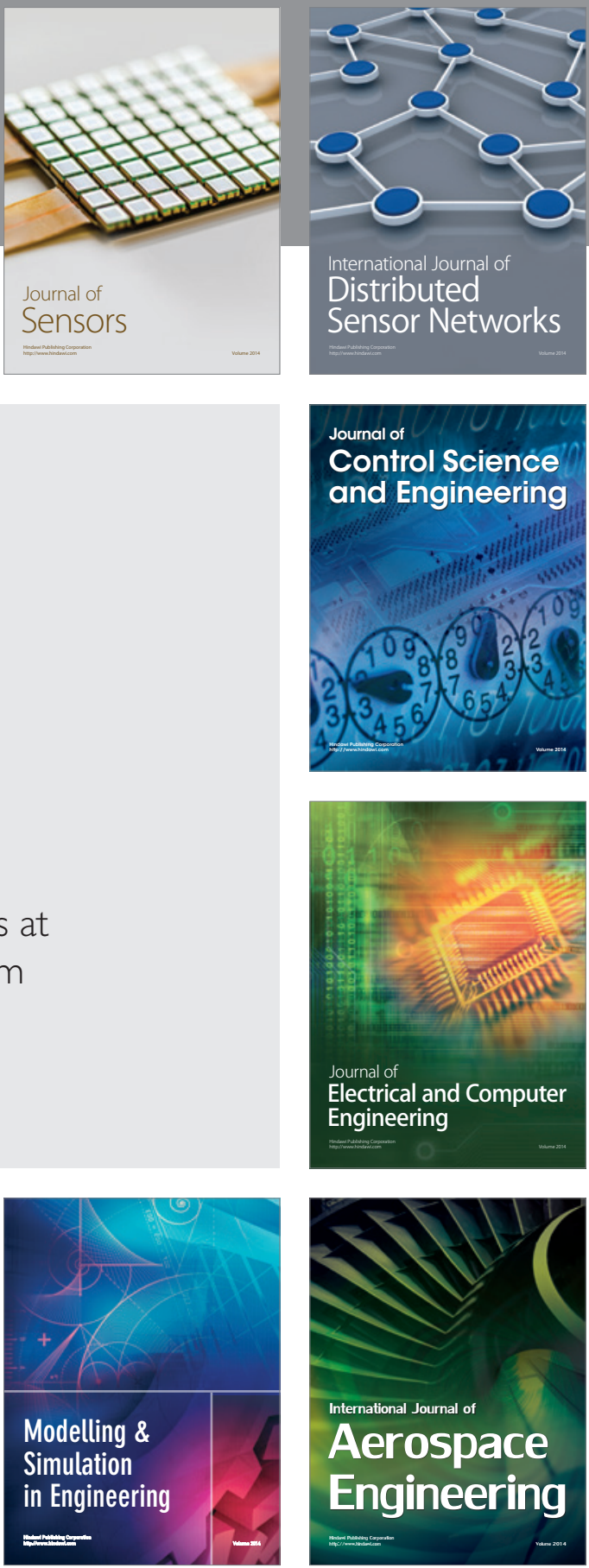

Journal of

Control Science

and Engineering
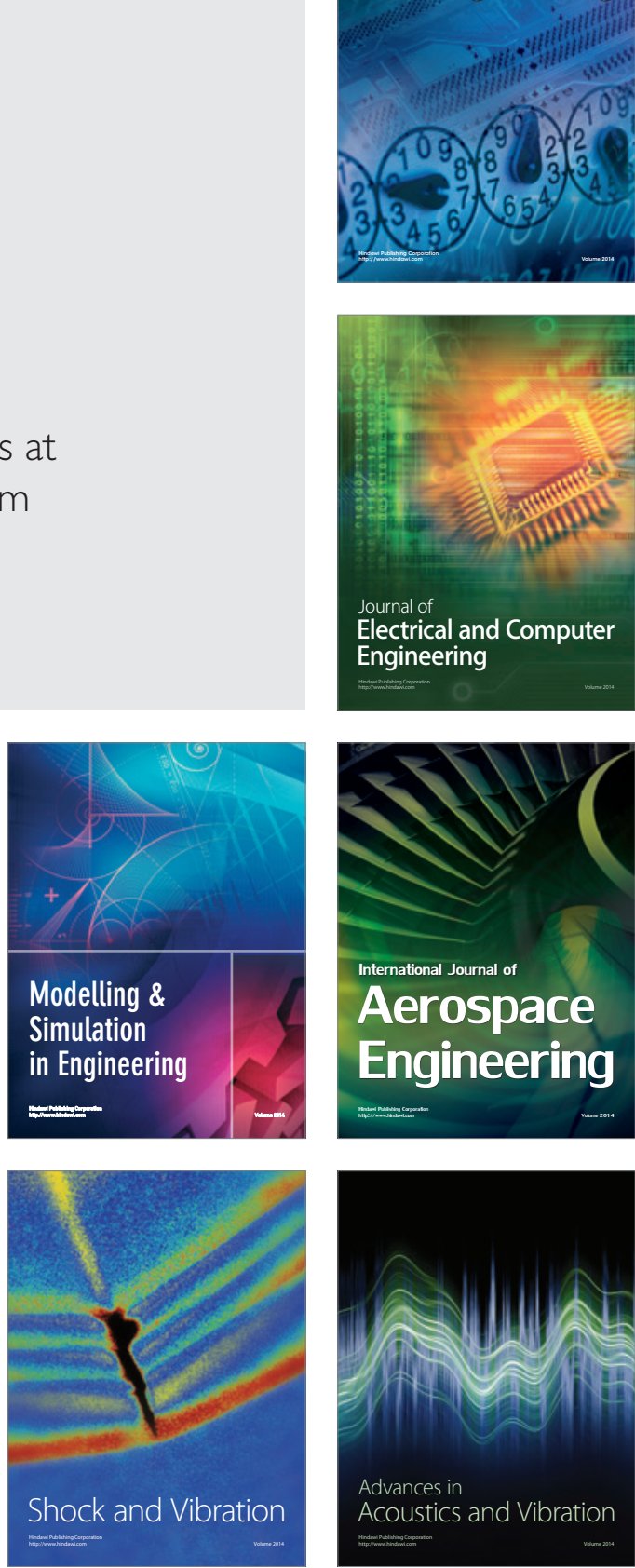